\title{
Improvement of Carrier Phase Tracking Based on a Joint Vector Architecture
}

\author{
Shaohua Chen and Yang Gao \\ Department of Geomatics Engineering, University of Calgary, Calgary, AB, Canada \\ Correspondence should be addressed to Shaohua Chen; shaohche@ucalgary.ca \\ Received 30 April 2017; Revised 21 June 2017; Accepted 28 June 2017; Published 30 July 2017 \\ Academic Editor: Linda L. Vahala \\ Copyright (C) 2017 Shaohua Chen and Yang Gao. This is an open access article distributed under the Creative Commons Attribution \\ License, which permits unrestricted use, distribution, and reproduction in any medium, provided the original work is properly cited. \\ Carrier phase measurements are essential to high precision positioning. Usually, the carrier phase measurements are generated from \\ the phase lock loop in a conventional Global Navigation Satellite System (GNSS) receiver. However there is a dilemma problem to \\ the design of the loop parameters in a conventional tracking loop. To address this problem and improve the carrier phase tracking \\ sensitivity, a carrier phase tracking method based on a joint vector architecture is proposed. The joint vector architecture contains \\ a common loop based on extended Kalman filter to track the common dynamics of the different channels and the individual loops \\ for each channel to track the satellite specific dynamics. The transfer function model of the proposed architecture is derived. The \\ proposed method and the conventional scalar carrier phase tracking are tested with a high quality simulator. The test results indicate \\ that carrier phase measurements of satellites start to show cycle slips using the proposed method when carrier noise ratio is equal \\ to and below $15 \mathrm{~dB}-\mathrm{Hz}$ instead of $21 \mathrm{~dB}-\mathrm{Hz}$ with using the conventional phase tracking loop. Since the joint vector based tracking \\ loops jointly process the signals of all available satellites, the potential interchannel influence between different satellites is also \\ investigated.
}

\section{Introduction}

Carrier phase is tracked by the phase lock loop (PLL) in a conventional GNSS receiver. Compared to frequency lock loop (FLL) and code lock loop (DLL), PLL is the weakest component which is sensitive to the noise and dynamics. However, to have usable carrier phase measurements, carrier phase tracking must be maintained. There are three error sources in carrier phase tracking [1]. One is the thermal noise. The second one is the oscillator noise which includes the vibration-induced noise, Allan Deviation oscillator phase noise, and $g$-sensitivity stress error. The third one is the dynamic stress error. In this paper we will focus on the analysis of the carrier tracking loop performance with oscillator noise and the thermal noise. To design a tracking loop, the loop bandwidth is a key parameter. There is also a dilemma situation to deal with in the bandwidth design [2]. The bandwidth, for instance, should be narrowed in order to decrease the thermal noise error but narrowing the bandwidth will increase the oscillator noise error and dynamic stress error.
With those challenges in mind, this paper will design a tracking architecture to address the problems.

Except the thermal noise, the oscillator and dynamics stress are dominated by the receiver clock and dynamics, and the satellite dynamics will be compensated for using ephemeris. The receiver oscillator and dynamic stress are common for different channels. If the common error can be estimated before the correlator for individual channels, the bandwidth of channel loop can be reduced without increasing the common error. One idea to handle these common errors is to use vector tracking. Leimer and Kohli [3] proposed a phase noise mitigation method which first used all available satellites to estimate the oscillator phase noise and then remove this oscillator error before the correlator. However they did not consider the common dynamics influence. Zhodzishsky et al. [4] proposed a Co-Op architecture for phase tracking based on the least square (LS) method. The Co-Op tracking architecture contains a common highbandwidth loop besides the conventional individual loop. However LS method is usually based on fixed bandwidth 
and is not flexible for different user modes. Based on CoOp tracking, multifrequency and multisatellite VPLL (MCVPLL) method was introduced by Henkel et al. [5] to eliminate the local filters for phase tracking for improved robustness. Giger et al. [6,7] proposed a MC-VPLL based on Kalman filter which includes many states to estimate. A mathematical model of Co-Op tracking is derived by Shafaati et al. [8] who have shown better performance in theory but no real data was processed.

In this paper, a carrier phase tracking approach based on a joint vector architecture is proposed for GNSS receivers. The proposed joint vector tracking method adds a common loop based on extended Kalman filter (EKF) to estimate the common error for different channels. Compared to the existing architectures mentioned above, the proposed method is able to take the advantage of EKF's flexibility and Co-Op tracking's robustness with less process states. A test with a high quality simulator shows that the proposed method can improve the sensitivity of carrier phase tracking by $6 \mathrm{~dB}$. Since the common loop combines all channels, the interchannel influence between different satellites is also investigated. The impact of the channel with lower carrier noise ratios (CNR) on other channels is especially provided. The test result shows that a lower CNR satellite can impose more noise on a higher CNR satellite.

The remainder of the paper is organized as follows. The methodology of the joint VPLL tracking architecture and the theoretical derivations are provided in Section 2. The test and performance comparison of the conventional scalar PLL and the proposed joint VPLL are given in Section 3. The interinfluence between different satellites with the joint vector tracking is analyzed in Section 4. Finally, some conclusions are drawn in Section 5.

\section{Joint Vector Phase Lock Loop}

In this section, the architecture of the joint VPLL is introduced firstly. The transfer function model based on the joint vector architecture is then derived. The implementation of the proposed joint VPLL is finally described.

2.1. Joint Vector Phase Lock Loop Architecture. The architecture of a joint vector phase lock loop (VPLL) is shown in Figure 1 in which the joint VPLL is implemented using an extended Kalman filter. The radio frequency (RF) signal is received by the antenna and then processed by the front-end which prefilters the RF signal and downconverts and samples the RF signal to Intermediate Frequency (IF) data.

The IF data is received and processed by the baseband algorithms to generate measurements including carrier phase, pseudorange, and Doppler. The baseband algorithms usually include acquisition and tracking. The fine synchronization of the input signal with the local replica is made by the tracking function which includes DLL, FLL, and PLL. In this paper, DLL is first-order loop aided by the PLL while FLL is not used. In the proposed joint VPLL, we add a vector phase lock loop in addition to the conventional PLLs dedicated to individual channels as shown in Figure 1. The vector loop uses

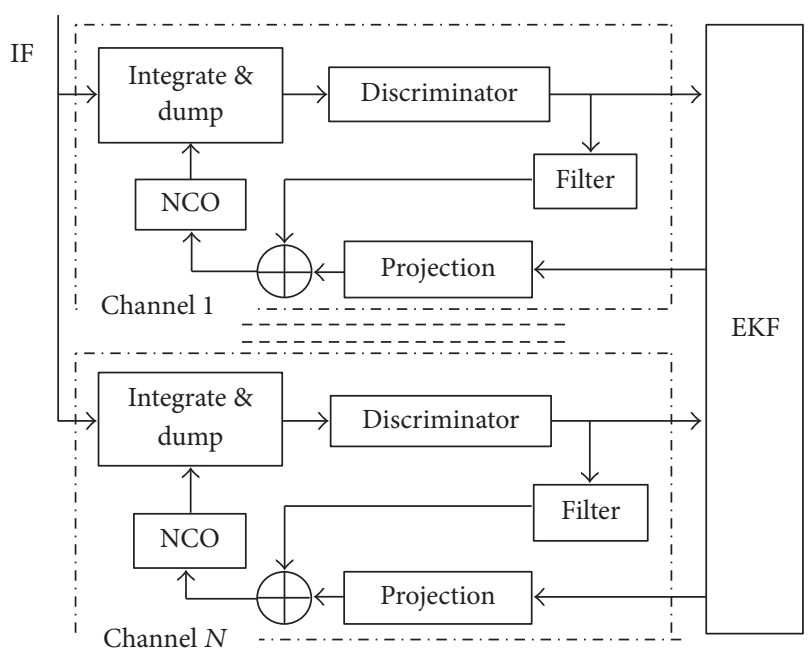

FIGURE 1: The joint VPLL architecture for carrier phase tracking.

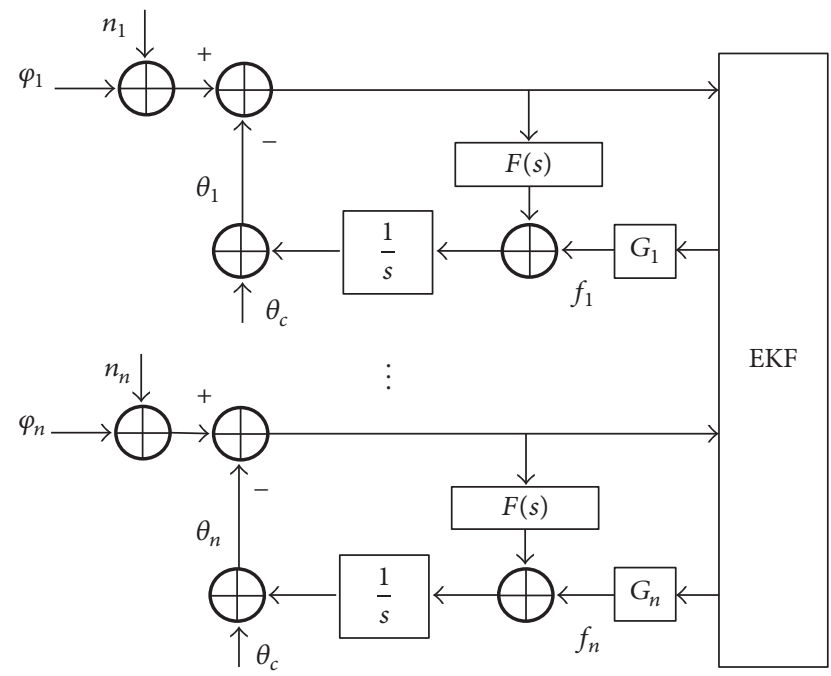

FIGURE 2: Transfer function models of the joint VPLL architecture.

the outputs of all channels discriminator as measurements to estimate the position and clock bias prediction error in the EKF block. The estimated states are used to feed back to the NCO through a projection block. Except the common loop, the conventional loops, each dedicated to a specific satellite, are conserved to track the channel-dependent dynamics. Second-order PLL is used in this research.

2.2. Transfer Function Models for the Joint VPLL. Figure 2 shows the transfer function (TF) model of the joint VPLL architecture. The TF model is a Laplace transform of the joint VPLL. The states of EKF are the position and oscillator prediction errors. There are some assumptions in the TF models. Firstly, the discriminator is assumed as linear subtraction. Secondly, the thermal noise includes all the effects, for example, the atmosphere, front-end, and antenna. 
The TF model of the common loop based on EKF can be written as follows [9]:

$$
\vec{f}=\left(I_{n \times n}+\frac{G_{n \times 4} K_{4 \times n}}{s}\right)^{-1} G_{n \times 4} K_{4 \times n}(\vec{\varphi}+\vec{n}-\vec{\theta}) .
$$

For all channels, the TF model is

$$
\vec{\theta}=[F(s)(\vec{\varphi}+\vec{n}-\vec{\theta})+\vec{f}] \frac{1}{s}+\theta_{c} I_{n \times 1} .
$$

Substitute (1) to (2) and get the TF model for all channels:

$$
\begin{aligned}
\vec{\theta}= & \left(F(s) I_{n \times n}+\left(I_{n \times n}+\frac{G_{n \times 4} K_{4 \times n}}{s}\right)^{-1} G_{n \times 4} K_{4 \times n}\right) \\
& \cdot(\vec{\varphi}+\vec{n}-\vec{\theta}) \frac{1}{s}+\theta_{c} I_{n \times 1} .
\end{aligned}
$$

For an individual channel, the TF model is

$$
\theta_{i}=\left[F(s)\left(\varphi_{i}+n_{i}-\theta_{i}\right)+f_{i}\right] \frac{1}{s}+\theta_{c},
$$

where $f_{i}$ is the output from the common filter for the $i$ th channel which is calculated based on all available channels in the EKF as described in (1).

From (3), we can get $\vec{\theta}$ as

$$
\begin{aligned}
\vec{\theta}= & {\left[I_{n \times n}+\frac{1}{s}\left(F(s) I_{n \times n}\right.\right.} \\
& \left.\left.+\left(I_{n \times n}+\frac{G_{n \times 4} K_{4 \times n}}{s}\right)^{-1} G_{n \times 4} K_{4 \times n}\right)\right]^{-1}\left\{\left[F(s) I_{n \times n}\right.\right. \\
& \left.+\left(I_{n \times n}+\frac{G_{n \times 4} K_{4 \times n}}{s}\right)^{-1} G_{n \times 4} K_{4 \times n}\right](\vec{\varphi}+\vec{n}) \\
& \left.+\theta_{c} I_{n \times 1}\right\},
\end{aligned}
$$

where $\vec{\varphi}$ is the input signal carrier phase vector including all channels: $\vec{\varphi}=\left[\begin{array}{llll}\varphi_{1} & \varphi_{2} & \cdots & \varphi_{n}\end{array}\right]^{T} ; \vec{\theta}$ is a replica of the local signal carrier phase vector including all channels: $\vec{\theta}=$ $\left[\begin{array}{llll}\theta_{1} & \theta_{2} & \cdots & \theta_{n}\end{array}\right]^{T} ; \vec{n}$ is the noise vector for all channels: $\vec{n}=$ $\left[\begin{array}{llll}n_{1} & n_{2} & \cdots & n_{n}\end{array}\right]^{T} ; \vec{f}$ is the common loop output vector for all channels: $\vec{f}=\left[\begin{array}{llll}f_{1} & f_{2} & \cdots & f_{n}\end{array}\right]^{T} ; I_{n \times n}$ is an $n \times n$ identity matrix; $K_{4 \times n}$ is the Kalman gain; $G_{n \times 4}$ is the geometry matrix; $\theta_{c}$ is the local oscillator phase noise; $n_{i}$ is the thermal noise in the $i$ th channel; $F(s)$ is the individual local filter transfer function.

For an individual channel in (4), the output of the NCO, $\theta_{i}$ is not only related to the channel's inputs such as $\varphi_{i}$ and $n_{i}$, but also related to $f_{i}$ which is related to all available channels in the EKF. In (5), there are two components, namely, the input signal and thermal noise $(\vec{\varphi}+\vec{n})$ and the oscillator phase noise $\theta_{c}$. We therefore can separate (5) into the following two equations:

$$
\begin{aligned}
\vec{\theta}_{s} & =\left[I_{n \times n}+\frac{1}{s}\left(F(s) I_{n \times n}\right.\right. \\
& \left.\left.+\left(I_{n \times n}+\frac{G_{n \times 4} K_{4 \times n}}{s}\right)^{-1} G_{n \times 4} K_{4 \times n}\right)\right]^{-1}\left[F(s) I_{n \times n}\right. \\
& \left.+\left(I_{n \times n}+\frac{G_{n \times 4} K_{4 \times n}}{s}\right)^{-1} G_{n \times 4} K_{4 \times n}\right](\vec{\varphi}+\vec{n}), \\
\vec{\theta}_{o} & =\left[I_{n \times n}+\frac{1}{s}\left(F(s) I_{n \times n}\right.\right. \\
& \left.\left.+\left(I_{n \times n}+\frac{G_{n \times 4} K_{4 \times n}}{s}\right)^{-1} G_{n \times 4} K_{4 \times n}\right)\right]^{-1} \theta_{c} I_{n \times 1},
\end{aligned}
$$

where $\vec{\theta}_{s}$ is the NCO output related to the input signal including the thermal noise; $\vec{\theta}_{o}$ is the NCO output related to the oscillator noise.

From (7), it can be seen that the oscillator phase noise is not only processed by the local filter but also by the EKF. If the EKF can estimate the oscillator error which is common to all channels, the design of the local filter $F(s)$ is then not limited by the oscillator noise. Similar to the oscillator noise shown in (6), the input signal and thermal noise are processed jointly by the EKF. The dynamic stress error can be estimated jointly by the joint VPLL. There are four states in the EKF. If the measurements of the EKF are more than four, then the variance of the dynamic stress error and oscillator noise error will be decreased which is similar to a vector delay lock loop (VDLL) [10,11]. If the error of the dynamic stress and oscillator noise can be estimated more precisely, the bandwidth of the local filter can be decreased which subsequently decreases the thermal noise error. In the optimal state, the dynamic stress error and the oscillator phase noise error could be estimated perfectly by the EKF. This allows the local filter $F(s)$ to be optimized according to the thermal noise which contains mainly channel-based error.

Although the dynamic stress error and oscillator phase noise error can be decreased using the joint VPLL, the joint tracking method will make the errors in one channel spread to other channels. Usually the thermal noise error is derived from the single channel in a conventional receiver. However for the joint VPLL, except the thermal noise error from the channel itself, the thermal noise error in this channel should include the errors generated from the common loop.

As we see in (6) and (7), the transfer function includes a matrix inverse which is related to all tracked satellites; it is very hard to derive the equivalent bandwidth in theory. So we use a simulator to assess the performance of the proposed VPLL. The focus will be on stationary receivers, since this will imply that thermal noise and oscillator phase noise are the main error sources. The dynamics induced by the satellite is calculated and compensated using ephemeris and user 
location. In the following section, the implementation of the joint VPLL is described in detail.

2.3. Implementation of the Joint VPLL. Based on the software receiver [12], the joint VPLL architecture is implemented. The individual channel of the joint VPLL is the same with the conventional PLL, although the loop parameters are different. For the EKF of the joint VPLL, the states, system model, and measurement model are all described below.

The state vector of the EKF is $x=\left[\begin{array}{llll}\delta x & \delta y & \delta z & \delta t\end{array}\right]^{T}$ which includes the errors in prediction of the change of the receiver position in the ECEF (Earth-Centered, Earth-Fixed) coordinate systems and the clock bias.

The system model is

$$
x_{k}=A x_{k-1}+w_{k-1},
$$

where $A$ is the transition matrix: $A=I_{4 \times 4}$ and $I_{4 \times 4}$ is an identity matrix; $w_{k}=\left[\begin{array}{llll}w_{x} & w_{y} & w_{z} & w_{t}\end{array}\right]^{T}$ represent the process noises in the three different coordinate directions and the oscillator induced noise, respectively. For the oscillator phase noise, here we consider the Allan deviation oscillator phase noise since the front-end is static. So the variance of the oscillator phase noise with Allan variance parameters can be calculated as follows [13]:

$$
\sigma_{t}^{2}=\frac{h_{0}}{2} \Delta t+\frac{2}{3} \pi^{2} h_{-2} \Delta t^{3},
$$

where $h_{0}$ is white frequency noise and $h_{-2}$ is random walk frequency noise; $h_{0}=2 \times 10^{-19}$ and $h_{-2}=2 \times 10^{-20}$ are for low quality temperature compensated crystal oscillator (TCXO) $[13,14]$. Then $\sigma_{t}^{2}=1.8 \times 10^{-4}$ in units of square meter for coherent integration time as $20 \mathrm{~ms}$. However, the precise Allan variance coefficients of the oscillator used in the test are unknown; we have test different values of $\sigma_{t}^{2}$ and set $\sigma_{t}^{2}=10 \times 10^{-4}$ in the following test. For the covariance matrix of the dynamic noise, a value of $1 \times 10^{-12} \mathrm{~m}^{2}$ for $\sigma_{x}^{2}, \sigma_{y}^{2}$, and $\sigma_{z}^{2}$ is used in the following test.

The measurement model is

$$
z_{k}=G x_{k}+v_{k},
$$

where $z=\left[\begin{array}{lll}\Delta \emptyset_{1} & \cdots & \Delta \emptyset_{N}\end{array}\right]^{T}$ is a $N \times 1$ vector which includes $N$ satellite phase error outputs from the phase discriminators; $G$ is the design matrix composed of slowly changing unit vectors dependent on the relative positions of the receiver and the satellites; $v=\left[\begin{array}{lll}v_{1} & \cdots & v_{N}\end{array}\right]^{T}$ represent the measurement noise which is assumed to be uncorrelated between different channels. The design matrix $G$ is

$$
G=\left[\begin{array}{cccc}
\alpha_{x}^{1} & \alpha_{y}^{1} & \alpha_{z}^{1} & -1 \\
\vdots & \vdots & \vdots & \vdots \\
\alpha_{x}^{i} & \alpha_{y}^{i} & \alpha_{z}^{i} & -1 \\
\vdots & \vdots & \vdots & \vdots \\
\alpha_{x}^{n} & \alpha_{y}^{n} & \alpha_{z}^{n} & -1
\end{array}\right],
$$

where $\alpha_{x}^{i}, \alpha_{y}^{i}$, and $\alpha_{z}^{i}$ are the line of sight unit vectors from the receiver's estimated position to the $i$ th satellite's position. The Kalman gain matrix $K$ in (1) can be calculated as

$$
K=P^{-} G^{T}\left(G P^{-} G^{T}+R\right)^{-1},
$$

where $P^{-}$is the covariance matrix of the predicted estimation error and $R$ is the covariance of the measurements noise. Since the measurements of different channels are uncorrelated, $R$ is a diagonal matrix shown as follows:

$$
R=\left[\begin{array}{ccc}
\sigma_{1}^{2} & \cdots & 0 \\
\vdots & \ddots & \vdots \\
0 & \cdots & \sigma_{n}^{2}
\end{array}\right],
$$

where $\sigma_{i}^{2}$ is the carrier phase discriminator output noise variance of the $i$ th satellite. An arctangent is used as discriminator. However the gain of an arctangent discriminator will be quickly decreased with decreasing SNR after the SNR is below $10 \mathrm{~dB}$. The process gain of the discriminator can be compensated as described in Curran [15], although the variance of the discriminator output will be increased when the process gain is adjusted. The variance of the discriminator output can be calculated according to Curran [15] as

$$
\sigma_{i}^{2} \approx \frac{\left(e^{-c_{1} \sqrt{\mathrm{SNR}}}+1\right) \operatorname{erf}\left(c_{2} \mathrm{SNR}\right)}{\mathrm{SNR}},
$$

where $c_{1}=0.8046$ and $c_{2}=0.3977$; SNR is the signal to noise ratio which can be derived from the CNR. CNR is calculated using the moments method [16].

\section{Test and Performance Comparison}

3.1. Test Description. A Spirent 8000 simulator is used to test the performance of the joint VPLL and compare to the performance using the conventional PLL method. In this research, we focus on the sensitivity improvement using the joint vector based tracking architecture. To isolate other error sources, the test antenna is put in a static mode during the experiments and the dynamics induced by satellite is to be estimated and compensated using the satellite ephemeris. As a result, the carrier phase error mostly would come from the oscillator and thermal noise. IF data for about 6 minutes are collected from the simulator with a CNR of $47 \mathrm{~dB}-\mathrm{Hz}$ for all satellite channels. In the experiments, Gaussian white noises are added to the last 5 minutes of the data to adjust the CNR for the purpose of assessing the performance using different tracking methods. Except the original data set with $47 \mathrm{~dB}-\mathrm{Hz}$, we generate 17 data sets which show that CNR are from $45 \mathrm{~dB}$ $\mathrm{Hz}$ down to $13 \mathrm{~dB}-\mathrm{Hz}$ with $2 \mathrm{~dB}$ intervals.

The geometry of satellites during the test period is shown in Figure 3, with 11 tracked satellites. Since no multipath and atmosphere influence are simulated, the satellites with lower elevation angle can also be used to analyze the performance using different tracking methods.

In the test, the coherent integration time is set as $20 \mathrm{~ms}$ to improve the tracking sensitivity. The dynamics of the satellite 


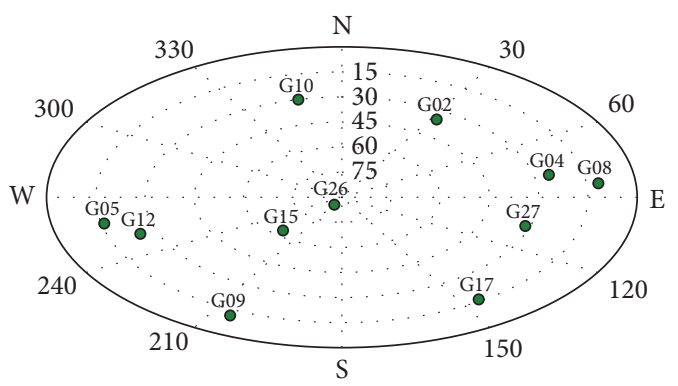

FIGURE 3: Geometry of satellites in the test.

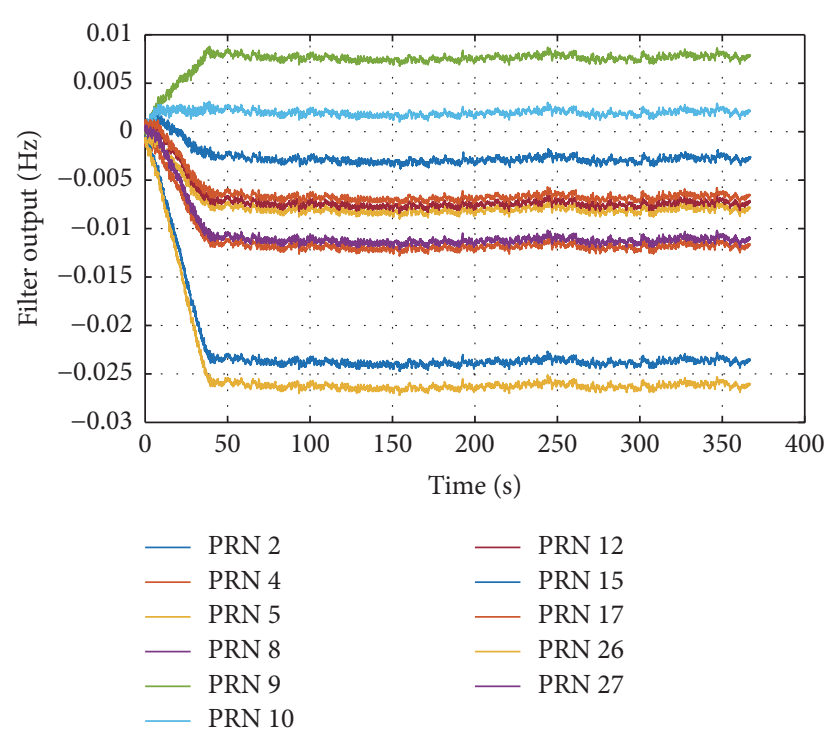

FIGURE 4: PLL filter output in the conventional GNSS receiver.

is compensated using the ephemeris and the receiver location information. Figure 4 shows the PLL filter output when the satellite dynamics is compensated for the original data with $47 \mathrm{~dB}-\mathrm{Hz}$.

In Figure 4, it is obvious that the PLL filter output does not change much after the satellite dynamics are compensated at the time of 40th second. After the compensation of satellite dynamics, the oscillator phase noise and thermal noise become the main factors that affect the carrier phase tracking.

In the test, we use the original $47 \mathrm{~dB}-\mathrm{Hz}$ data to generate the carrier phase measurements $\emptyset_{47 \mathrm{~dB}-\mathrm{Hz}}$ as reference. Then the other data sets with different CNR are processed to generate the carrier phase measurements $\emptyset_{\mathrm{CNR}}$. The indication of the performance to compare the two methods is the single difference of carrier phase measurements between $\emptyset_{47 \mathrm{~dB}-\mathrm{Hz}}$ and $\emptyset_{\mathrm{CNR}}$ which is shown as follows:

$$
\emptyset_{\text {diff }}=\emptyset_{\mathrm{CNR}}-\emptyset_{47 \mathrm{~dB}-\mathrm{Hz}} \text {. }
$$

$\emptyset_{\text {diff }}$ should show white noise since we only add Gaussian white noises to the original data set. However since we use different bandwidths to process the data sets, $\emptyset_{\text {diff }}$ will not show white noise and the standard deviation (STD) of $\emptyset_{\text {diff }}$ will not be able to be used as performance indicator since the

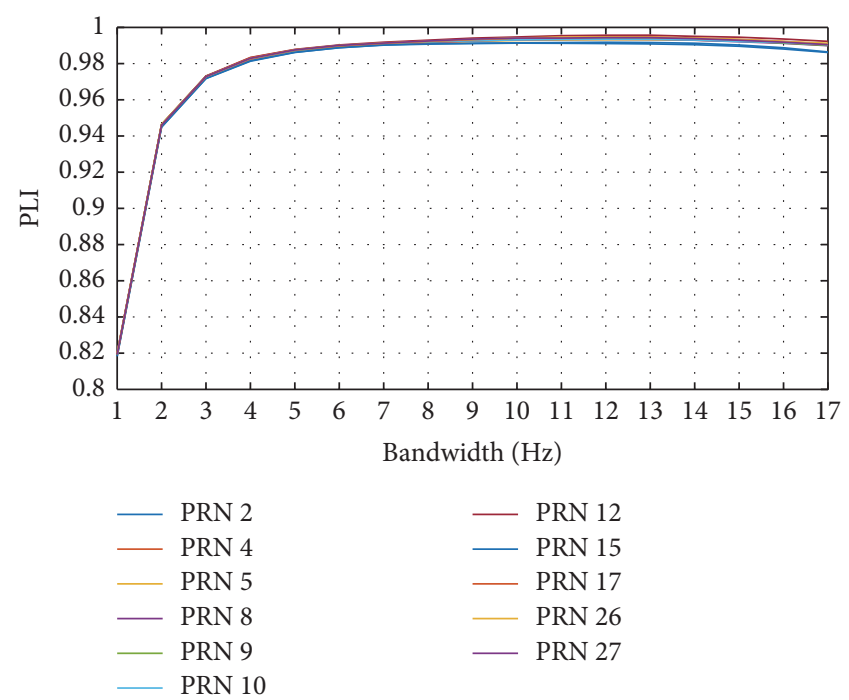

FIGURE 5: Mean of PLI under different bandwidth for the data set with $47 \mathrm{~dB}-\mathrm{Hz}$ data set.

STD depends on the bandwidth. In this paper, we check the cycle slips of $\emptyset_{\text {diff }}$ to indicate the performance of the tracking loop.

Firstly, to establish a reference for the test, we have tried different bandwidths to process the original data set to find the optimal bandwidth and the corresponding phase lock indicator (PLI) [2] under different bandwidths are shown in Figure 5.

In Figure 5, it can be seen that the PLI is greater than 0.96 when the bandwidth is above $4 \mathrm{~Hz}$. Since the product of bandwidth and integration time should be much less than 1 in order to ensure the stability of the loop [17], it is better to use a smaller bandwidth. However it is obvious that PLI values are significantly reduced when the bandwidth is reduced to $1 \mathrm{~Hz}$. To verify the influence of the oscillator, the PLI for different satellites with $1 \mathrm{~Hz}$ bandwidth is shown in Figure 6.

As shown in Figure 6, after the bandwidth is set to $1 \mathrm{~Hz}$ which starts at 50 seconds, the PLI values for all satellites are reduced because the oscillator has caused the same influence on all satellites. Considering all above influences, a bandwidth of $5 \mathrm{~Hz}$ will be applied to generate the reference carrier phase measurements.

3.2. Performance Comparison. An analysis on the single difference of carrier phase measurements indicates that cycle slips will be present when the CNR is equal to or less than $21 \mathrm{~dB}-\mathrm{Hz}$. Therefore, the data sets with $21 \mathrm{~dB}-\mathrm{Hz}$ and less CNR will be used in the following performance comparison between the conventional scalar tracking and the joint VPLL.

Given in Figure 7 are the single differences of carrier phase measurements using the conventional PLL method. Four satellites, namely, PRN 4, PRN 8, PRN 9, and PRN 15, have experienced cycle slips in their carrier phase measurements. Since the optimal bandwidth $3 \mathrm{~Hz}$ (obtained through testing different bandwidths) has been used, the result is considered to be the best performance using the conventional 


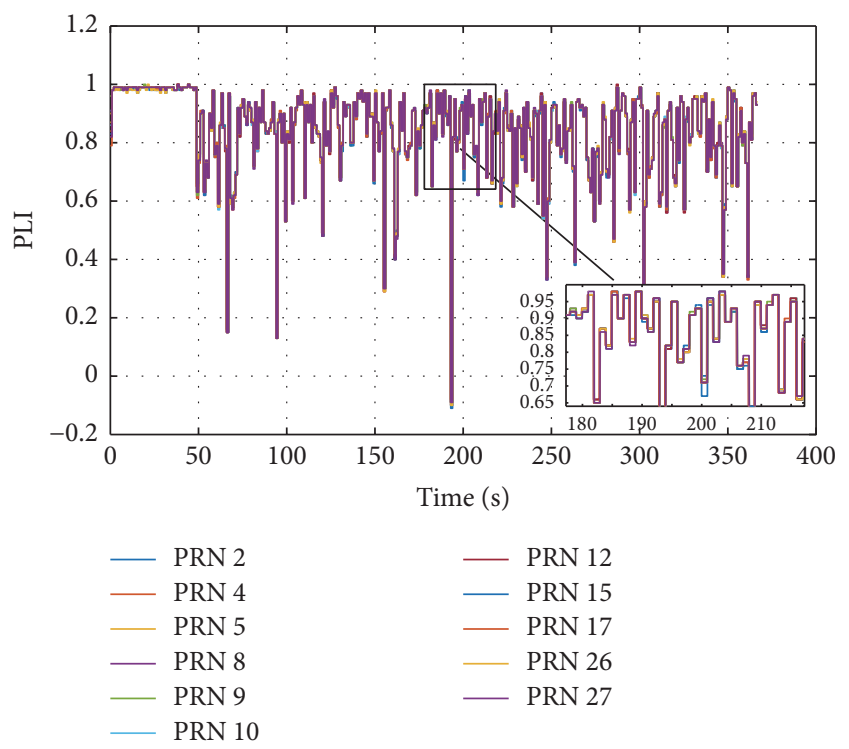

FIGURE 6: PLI for different satellites with $1 \mathrm{~Hz}$ bandwidth.

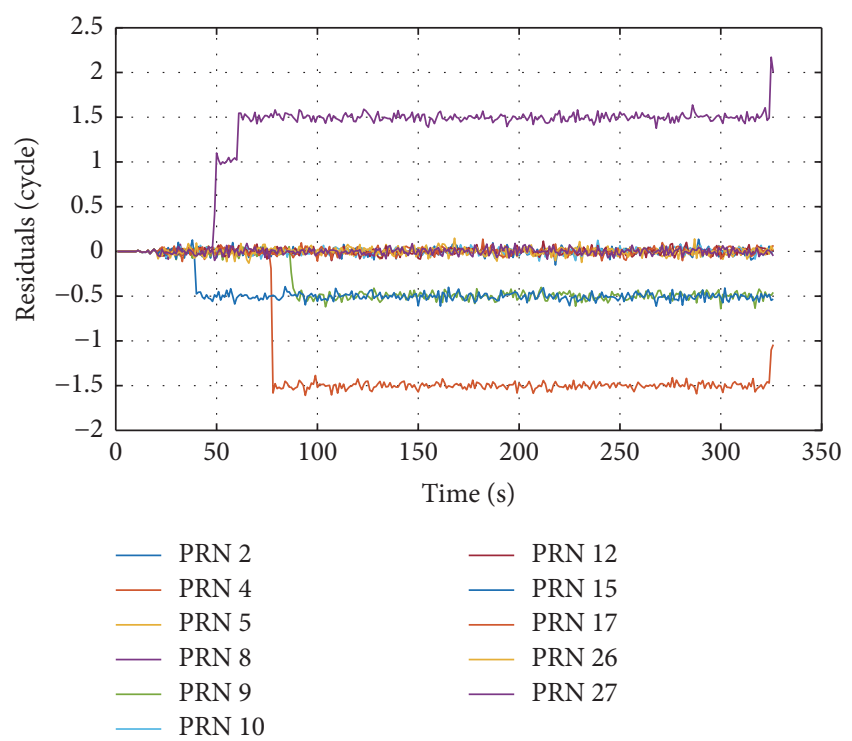

FIGURE 7: Single difference of carrier phase between $47 \mathrm{~dB}-\mathrm{Hz}$ and $21 \mathrm{~dB}-\mathrm{Hz}$ data sets with PLL.

PLL method. More cycle slips however would be expected when a less desired bandwidth is applied.

Shown in Figure 8 are the single differences of carrier phase measurements using the proposed joint VPLL method. The results indicate that no cycle slips are present for all satellites although the standard deviation of the carrier phase measurements is large. A comparison between Figure 8 and Figure 7 confirms that the proposed tracking method can provide more robust performance than the conventional PLL architecture.

Shown in Figures 9 and 10 are the results when the CNR is $19 \mathrm{~dB}-\mathrm{Hz}$, using the conventional PLL and the proposed joint VPLL methods, respectively. We see that all carrier phase measurements are experienced with cycle slips with the

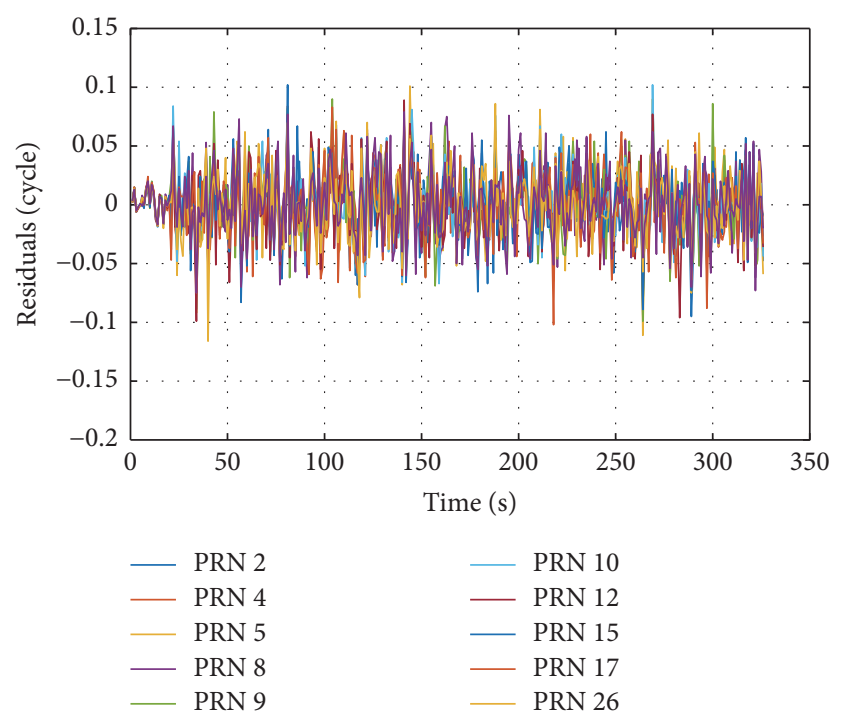

FIgURE 8: Single difference of carrier phase between $47 \mathrm{~dB}-\mathrm{Hz}$ and $21 \mathrm{~dB}-\mathrm{Hz}$ data sets with the joint VPLL.

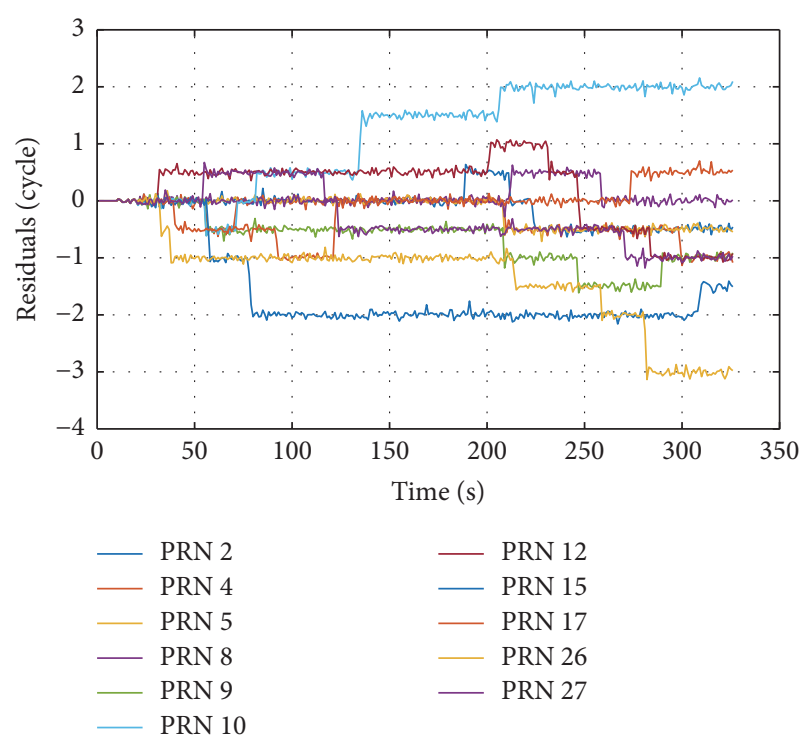

FIGURE 9: Single difference of carrier phase between $47 \mathrm{~dB}-\mathrm{Hz}$ and $19 \mathrm{~dB}-\mathrm{Hz}$ data sets with PLL.

conventional PLL method. However, there are no cycle slips for all satellites when using the proposed joint VPLL method.

Similar results are gotten when the CNR is $17 \mathrm{~dB}-\mathrm{Hz}$ and no cycle slips are observed using the joint VPLL but all satellites experience cycle slips with conventional PLL. However, it is worth mentioning that all satellite carrier phase measurements would experience cycle slips when the CNR of the data set is equal to or below $15 \mathrm{~dB}-\mathrm{Hz}$, even using the proposed method as shown in Figures 11 and 12. Nevertheless, it is still obvious that the proposed method shows that it would provide more robust performance compared to the conventional PLL method.

Based on the test results described above, the proposed joint VPLL architecture can improve the carrier phase 


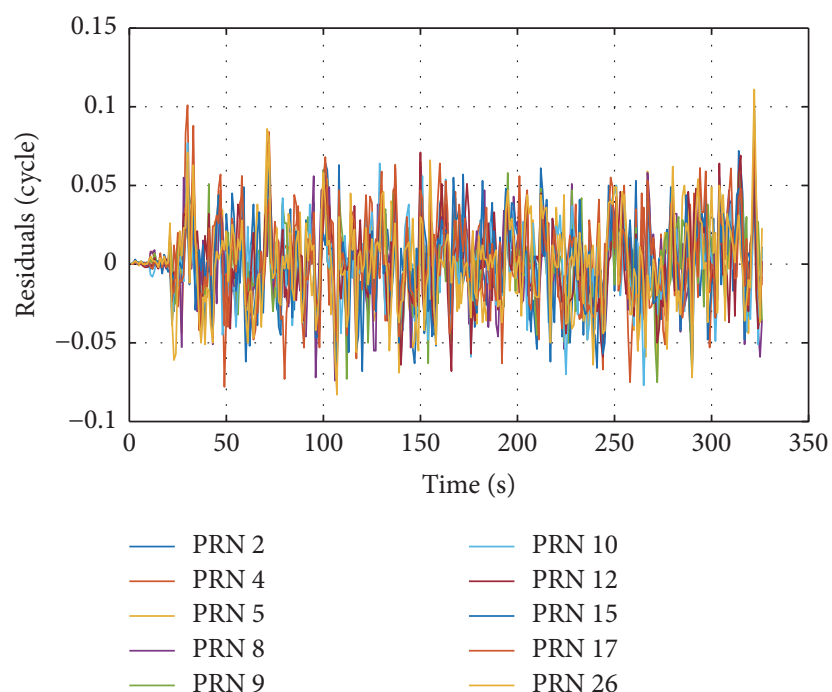

Figure 10: Single difference of carrier phase between $47 \mathrm{~dB}-\mathrm{Hz}$ and $19 \mathrm{~dB}-\mathrm{Hz}$ data sets with the joint VPLL.

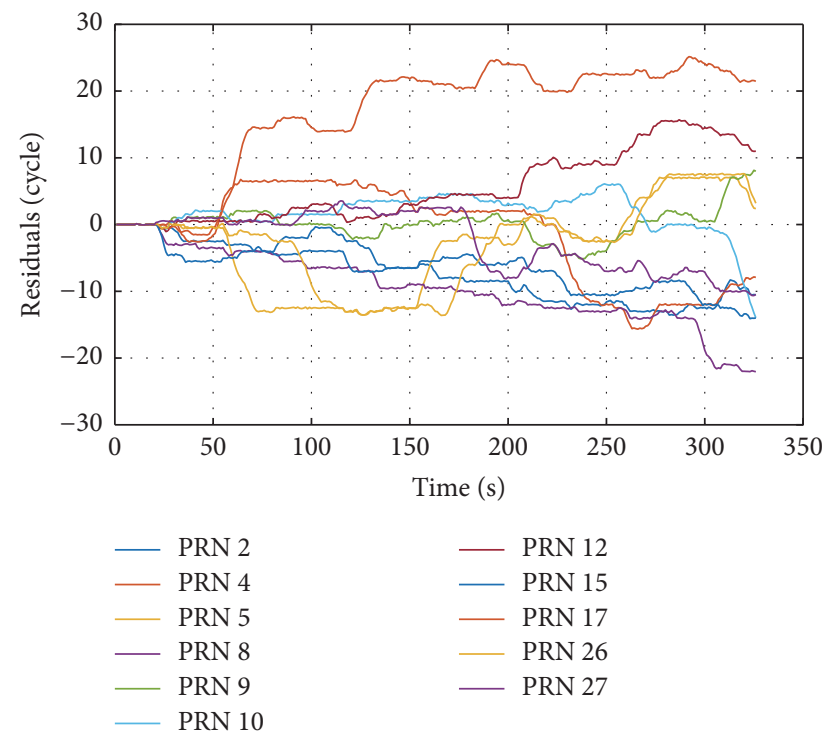

FIGURE 11: Single difference of carrier phase between $47 \mathrm{~dB}-\mathrm{Hz}$ and $15 \mathrm{~dB}-\mathrm{Hz}$ data sets with PLL.

tracking performance by about $6 \mathrm{~dB}$ when compared to the conventional PLL architecture. However, the interchannel influences between different satellites have to be accounted for using the vector based PLL especially when the channel with lower CNR affects the channels with higher CNR. This will be described in the next section.

\section{Interchannel Influence of Different Satellites in the Joint Vector Tracking}

To assess the interchannel influence, another data set is collected which is the same as the data described in the previous section except with a different CNR. In this data set, the CNR for satellite PRN27 is set to be $27 \mathrm{~dB}-\mathrm{Hz}$ while

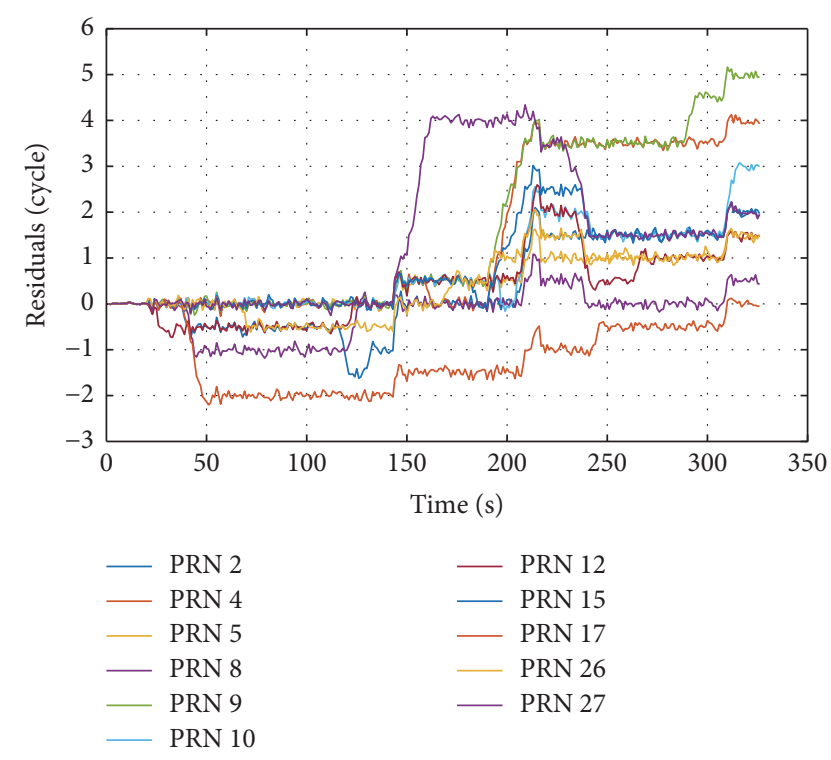

FIGURE 12: Single difference of carrier phase between $47 \mathrm{~dB}-\mathrm{Hz}$ and $15 \mathrm{~dB}-\mathrm{Hz}$ data sets with the joint VPLL.

other satellites are with $47 \mathrm{~dB}-\mathrm{Hz}$. We call this data set dataset $\mathrm{A}$ in the sequel. Gaussian white noise is added to dataset $A$ to adjust the CNR and generate data-set $B$ which shows $12 \mathrm{~dB}$ lower compared to data-set A. So in data-set B, CNR of satellite PRN 27 is $15 \mathrm{~dB}-\mathrm{Hz}$ and other satellites show $35 \mathrm{~dB}$ $\mathrm{Hz}$.

The carrier phase measurement of data-set $\mathrm{A}$ is set as reference. We use the same analysis method described in previous section to process data-set A and data-set B. However to explore the influence of the lower CNR satellites, two strategies are used to process the data-set B.

Strategy One. Using the joint VPLL method: set measurements variances for different satellites in the $R$ matrix are the same which means all the measurements variances are calculated based on $35 \mathrm{~dB}-\mathrm{Hz}$.

Strategy Two. Using the joint VPLL method: calculate measurement noise variance of satellite PRN 27 using $15 \mathrm{~dB}-\mathrm{Hz}$ and other satellites using $35 \mathrm{~dB}-\mathrm{Hz}$.

The result of single difference of carrier phase measurements between data-set A and data-set B with two strategies for PRN 27 is shown in Figure 13.

In Figure 13, the second strategy shows less cycle slips and better performance compared to the first strategy. The influence of PRN 27 for the joint VPLL is reduced in the second strategy since the covariance of PRN measurements is calculated using $15 \mathrm{~dB}-\mathrm{Hz}$ rather than $35 \mathrm{~dB}-\mathrm{Hz}$ compared to the first strategy. The single difference of carrier phase measurements for PRN 2 is shown in Figure 14.

With strategy two, satellite PRN 2 shows better performance than that using strategy one in Figure 14. Standard deviation of residuals for PRN 2 using strategy two is 0.0129 ; however strategy one shows 0.0165 . The lower CNR satellite can impose more noise on the higher CNR satellites due to 


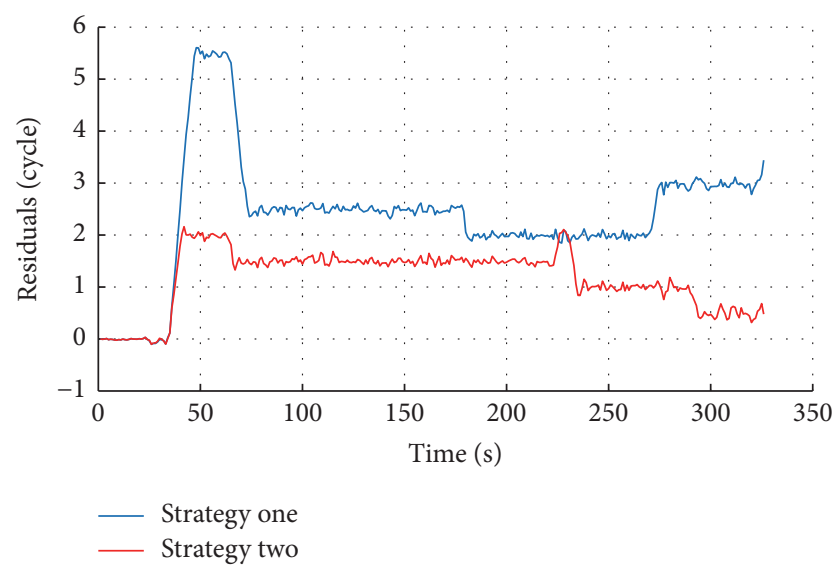

FIGURE 13: Residuals of satellite PRN 27 using two processing strategies.

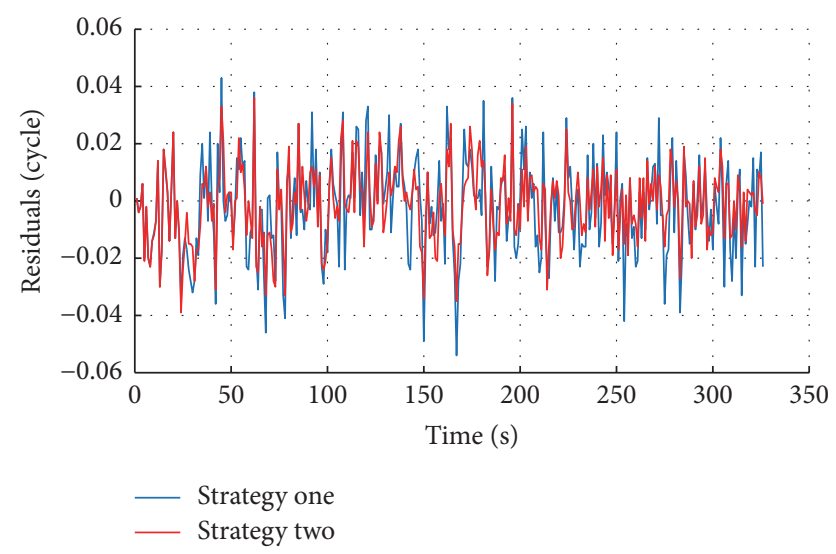

FIGURE 14: Residuals of satellite PRN 2 using two processing strategies.

the common loop. In the joint VPLL tracking, since $R$ matrix of EKF is calculated based on the CNR, the influence of lower CNR satellite can be reduced which is similar to strategy two.

\section{Conclusions}

In this paper, the influence of the oscillator in the GNSS receiver is analyzed. To address the dilemma problem of loop bandwidth design, a carrier phase tracking method based on a joint vector architecture is proposed. The transfer function model of the proposed method is derived and analyzed. Based on the same oscillator, we test different CNR through adding white noise on the IF data. With the IF data collected from simulator and the data sets with more white noise, the proposed method is proved to improve the carrier phase tracking sensitivity by around $6 \mathrm{~dB}$ when compared to the conventional scalar tracking which uses the optimal bandwidth during the test. Interchannel influence of the proposed method is also analyzed. Due to the joint tracking in the joint VPLL, the lower CNR satellite imposes more noise on the higher CNR satellite. However, as $R$ matrix of EKF in the joint VPLL is calculated based on CNR, the influence of the satellite with lower CNR can be reduced.

\section{Conflicts of Interest}

The authors declare that there are no conflicts of interest regarding the publication of this paper.

\section{Acknowledgments}

The first author is supported by the Alberta Innovates Technology Futures (AITF) through The Alberta Doctoral Awards for Chinese Students (ADACS) program. This work is also supported by Tecterra.

\section{References}

[1] PW. Ward, JW. Betz, and CJ. Hegarty, "Satellite signal acquisition, tracking, and data demodulation," in Understanding GPS principles and applications, E. Kaplan and C. Hegarty, Eds., pp. 153-241, Artech House, Pimlico, London, 2nd edition, 2006.

[2] J. J. Spilker Jr., P. Axelrad, B. W. Parkinson, and P. Enge, "Fundamentals of signal tracking theory," in Global Positioning System: Theory And Applications, B. W. Parkinson, J. J. Spilker, and P. Enge, Eds., vol. 1, pp. 245-327, American Institute of Aeronautics and Astronautics, Inc., Washington, DC, USA, 1996.

[3] D. Leimer and S. Kohli, "Receiver phase-noise mitigation," in Proceedings of the 11th International Technical Meeting of the Satellite Division of the Institute of Navigation, pp. 627-632, 1998.

[4] M. Zhodzishsky, S. Yudanov, V. Veitsel, and J. Ashjaee, "CoOp tracking for carrier phase," in Proceedings of the 11th International Technical Meeting of the Satellite Division of The Institute of Navigation, pp. 653-664, 1998.

[5] P. Henkel, K. Giger, and C. Günther, "Multifrequency, multisatellite vector phase-locked loop for robust carrier tracking," IEEE Journal on Selected Topics in Signal Processing, vol. 3, no. 4, pp. 674-681, 2009.

[6] K. Giger, P. Henkel, and C. Günther, "Multifrequency multisatellite carrier tracking. in," in Proceedings of the 4th Europ. Worksh. on GNSS Sig. and Sig. Proc, 2009.

[7] K. Giger and C. Günther, "Multisatellite tracking GNSS receivers in multipath environments," in Proceedings of the ION GNSS, pp. 1140-1151, September 2011.

[8] A. Shafaati, T. Lin, and G. Lachapelle, "Performance comparison of difference correlator and co-op tracking architectures under receiver clock instability," in Proceedings of the 28th International Technical Meeting of The Satellite Division of the Institute of Navigation, pp. 2887-2904, September 2015.

[9] S. Bhattacharyya and D. Gebre-Egziabher, "Development and validation of parametric models for vector tracking loops," Journal of the Institute of Navigation, vol. 57, no. 4, pp. 275-295, 2010.

[10] M. Lashley and DM. Bevly, "What are vector tracking loops and what are their benefits and drawbacks?" Inside GNSS, 2009, http://www.insidegnss.com/node/1458.

[11] T. Pany and B. Eissfeller, "Use of a vector delay lock loop receiver for GNSS signal power analysis in bad signal conditions," in Proceedings of the 2006 IEEE/ION Position, Location, and Navigation Symposium, pp. 893-903, San Diego, CA, USA, April 2006. 
[12] S. Chen and Y. Gao, "Implementation and performance analysis of an adaptive vector tracking loop utilizing precise satellite orbit/clock and atmospheric model," in Proceedings of the ION GNSS+, pp. 1174-1180, Tampa, FL, USA, 2015.

[13] RG. Brown and P. Y. C. Hwang, Introduction to Random Signals and Applied Kalman Filtering with Matlab Exercises, 4th edition, 2012.

[14] M. Lashley and D. M. Bevly, "Comparison in the performance of the vector delay/frequency lock loop and equivalent scalar tracking loops in dense foliage and urban canyon," in Proceedings of the the 24th International Technical Meeting of the Satellite Division of the Institute of Navigation, pp. 1786-2001, September 2011.

[15] J. T. Curran, "Enhancing weak-signal carrier phase tracking in GNSS receivers," International Journal of Navigation and Observation, vol. 2015, Article ID 295029, 15 pages, 2015.

[16] E. Falletti, M. Pini, and L. L. Presti, "Low complexity carrierto-noise ratio estimators for GNSS digital receivers," IEEE Transactions on Aerospace and Electronic Systems, vol. 47, no. 1, pp. 420-437, 2011.

[17] P. L. Kazemi, "Optimum digital filters for GNSS tracking loops," in Proceedings of the 21st International Technical Meeting of the Satellite Division of The Institute of Navigation, pp. 2304-2313, Savannah, GA, USA, September 2008. 


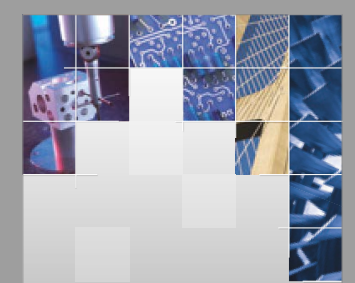

\section{Enfincering}
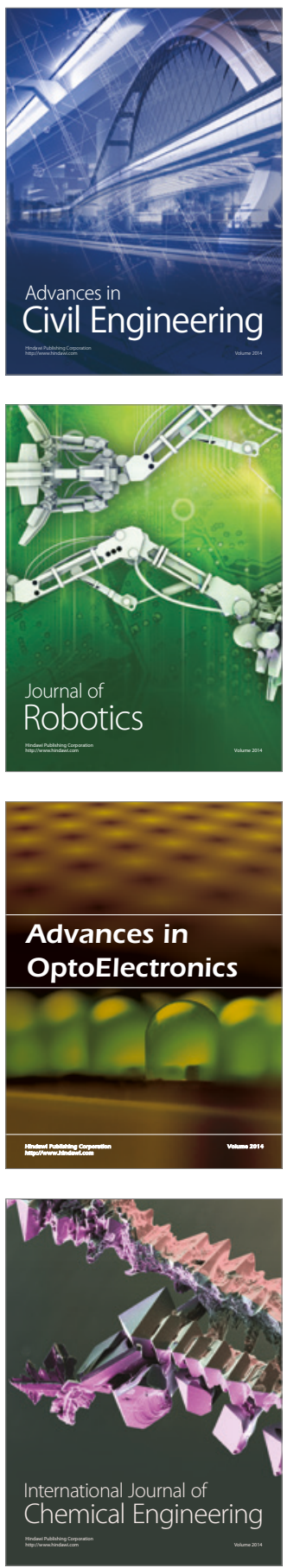

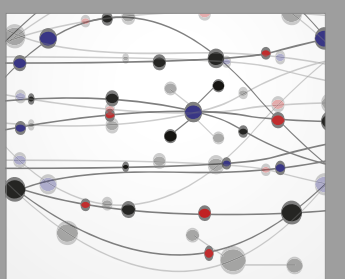

The Scientific World Journal

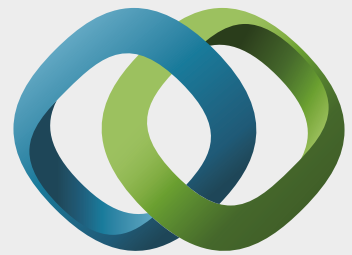

\section{Hindawi}

Submit your manuscripts at

https://www.hindawi.com
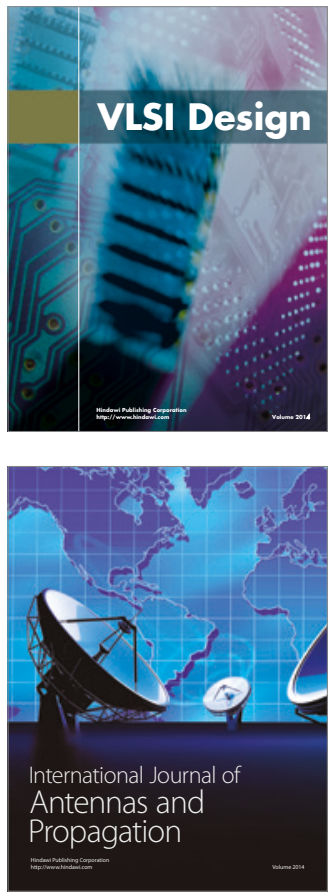

\section{Rotating}

Machinery
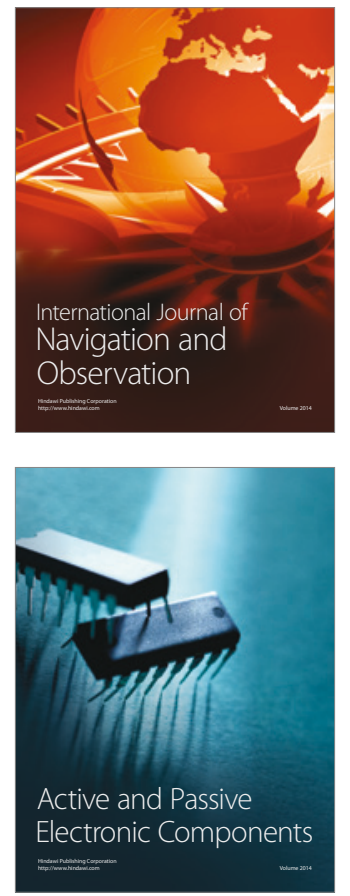
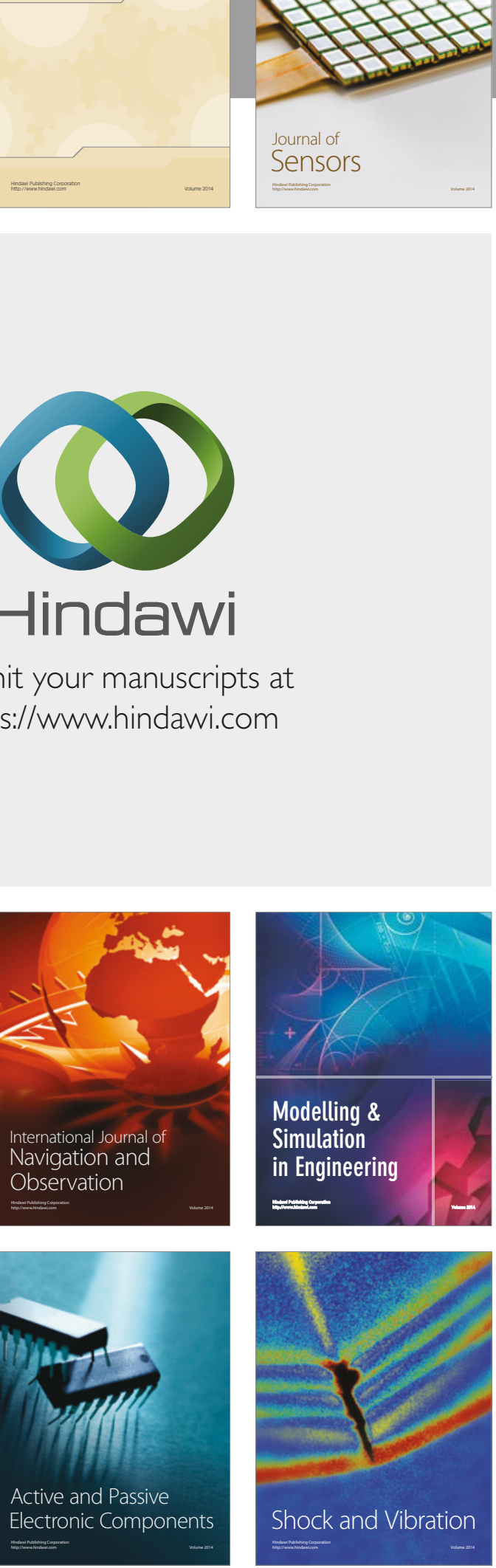
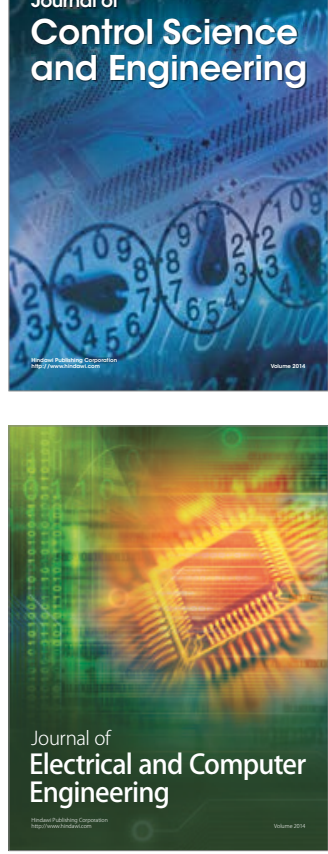

Distributed

Journal of

Control Science

and Engineering
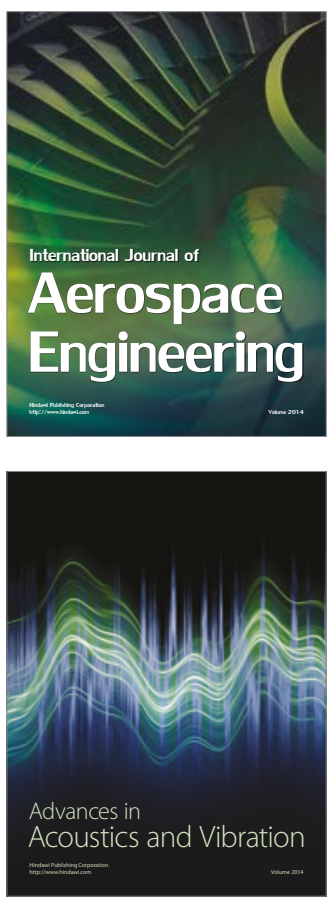

Sensor Networks 